\title{
Replacement manifolds: A method to uniformize semiclassical wave functions
}

\author{
Jiř́ Vaníček and Eric J. Heller \\ Department of Physics, Harvard University, Cambridge, Massachusetts 02138 \\ (Received 31 January 2001; revised manuscript received 2 May 2001; published 19 July 2001)
}

\begin{abstract}
We present a semiclassical technique that relies on replacing complicated classical manifold structure with simpler manifolds, which are then evaluated by the usual semiclassical rules. Under circumstances where the original manifold structure gives poor or useless results semiclassically the replacement manifolds can yield remarkable accuracy. We give several working examples to illustrate the theory presented here.
\end{abstract}

DOI: $10.1103 /$ PhysRevE.64.026215

PACS number(s): 05.45.Mt, 03.65.Sq, 34.50.Dy

\section{INTRODUCTION}

Semiclassical methods are based on classical mechanics; the relevant classical manifolds form the "skeleton" to which the wave function is attached. However, associating a semiclassical wave function with a classical manifold is problematic when enclosed loops in phase space have an area less than $\hbar$. If that happens, typically two or more stationary-phase points corresponding to distinct contributions to the semiclassical wave function are not well separated in phase. Consequently, the stationary-phase (SP) method, on which the semiclassical approximation is based, breaks down. Worse, in some important situations, such as the universal homoclinic oscillations associated with chaotic regions of phase space, the bad enclosed loops may be repeated many times in a small region [1].

Although the semiclassical wave function diverges near caustics lurking near the small area loops, the exact quantum wave function is of course well behaved. This is one manifestation of quantum smoothing over classical detail and we shall quantify below how this smoothing occurs. We suggest and test a new way of looking at the smoothing process in terms of "replacement manifolds," in which new, well behaved classical manifolds are substituted for the original badly behaved ones. One application of this approach is uniformizing a semiclassical wave function in the vicinity of the ubiquitous homoclinic oscillations of a chaotic system.

It is widely understood that very small changes in potentials or walls of billiards can have little effect on quantum eigenstates, but can drastically affect the classical manifold structure. This immediately implies a many-to-one relationship between the classical manifolds and quantum wave functions. That is, many different underlying classical manifold patterns correspond to the same wave function. Now, whatever the choice of manifold (amongst these equivalent forms) we can suppose an appropriate uniformization exists that gives the correct wave function. However, the nonuniformized, simple semiclassical limit for the various choices of manifolds can differ drastically in accuracy. It is this fact that we exploit in the present paper.

\section{STANDARD UNIFORMIZATION: THE AIRY FUNCTION}

The paradigm example of uniformization is the Airy function, i.e., the energy eigenfunction of the linear ramp poten- tial. We take the potential to be of the form

$$
V(q)=-\beta q .
$$

The Schrödinger equation is

$$
-\frac{\hbar^{2}}{2 m} \psi_{E}^{\prime \prime}(q)-(\beta q+E) \psi_{E}(q)=0 .
$$

After the shift

$$
\psi_{E}(q)=\psi_{0}(q+E / \beta)
$$

and scaling transformation

$$
u=\frac{m^{1 / 3} \beta^{1 / 3}}{\hbar^{2 / 3}} q \equiv \gamma q
$$

the Schrödinger equation reads

$$
\frac{1}{2} \Psi^{\prime \prime}(u)+u \Psi(u)=0
$$

with $\Psi(u)=\psi_{0}(q)$. Equation (5) is exactly solved by going to the momentum representation. We have

$$
\left(\frac{1}{2} p^{2}-i \frac{\partial}{\partial p}\right) \Phi(p)=0
$$

with solution (up to normalization)

$$
\Phi(p)=e^{-i p^{3} / 6} .
$$

Thus

$$
\begin{aligned}
\Psi(u) & =\frac{1}{\sqrt{2 \pi}} \int_{-\infty}^{\infty} e^{i p u} \Phi(p) d p \\
& =\frac{2}{\sqrt{2 \pi}} \int_{0}^{\infty} \cos \left(p^{3} / 6-p u\right) d p \\
& =2^{1 / 3} \sqrt{2 \pi} \operatorname{Ai}\left(-2^{1 / 3} u\right)
\end{aligned}
$$

and

$$
\psi(q) \propto \operatorname{Ai}\left(-2^{1 / 3} \gamma q\right)=\operatorname{Ai}\left(\frac{-2^{1 / 3} m^{1 / 3} \beta^{1 / 3}}{\hbar^{2 / 3}} q\right) .
$$




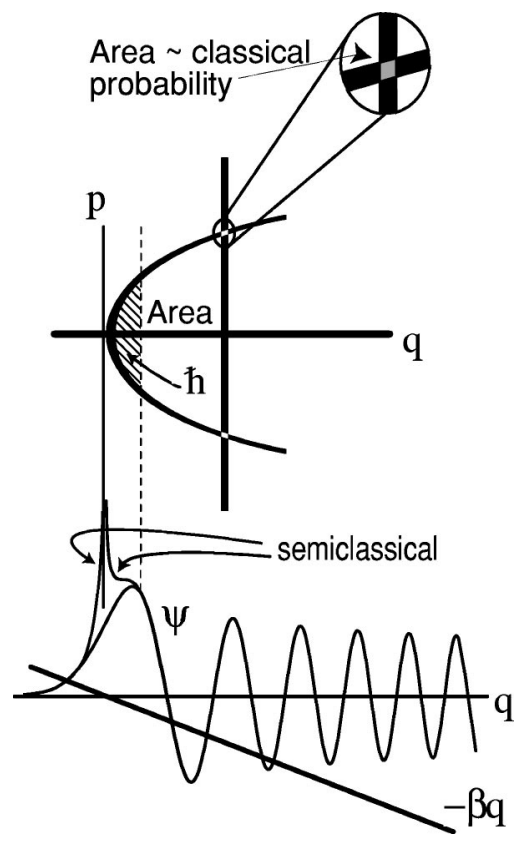

FIG. 1. Diagram showing the areas in phase space important to the semiclassical approximation to the Airy function.

The SP approximation for the momentum integral gives

$$
\begin{aligned}
\Psi(u) & \sim\left[\frac{1}{-i(-\sqrt{2 u})}\right]^{1 / 2} e^{-i(\sqrt{2 u})^{3} / 6+i \sqrt{2 u} u}+\text { c.c. } \\
& \sim \frac{2^{3 / 4}}{u^{1 / 4}} \cos \left(\frac{2}{3} \sqrt{2} u^{3 / 2}-\frac{\pi}{4}\right) .
\end{aligned}
$$

Noting that

$$
\int^{u} p\left(u^{\prime}\right) d u^{\prime}=\frac{(2 u)^{3 / 2}}{3}
$$

we get finally

$$
\Psi(u) \sim \frac{2}{\sqrt{p(u)}} \cos \left(\int^{u} p\left(u^{\prime}\right) d u^{\prime}-\frac{\pi}{4}\right) .
$$

This is the standard WKB form for the linear ramp potential.

Figure 1 illustrates the situation for the semiclassical position wave function, which becomes inaccurate when the area enclosed by the vertical $q$ line and the energy contour corresponding to the state $|E\rangle$ falls below Planck's constant. Upon entering the "bad" region, the original momentum integral may be substituted for the WKB result, giving a uniformization. We have an integral over "initial momentum," making this example a type of "initial value representation" (IVR), as discussed by Miller [2,3]. Of course in this case we know the result of the integral is an Airy function, but in general there may be integrals that are neither known analytically nor similar to ones that are.

In this paper we encounter more complex situations that are induced by nonlinear interactions, including chaos, reflection from corrugated surfaces, etc. We will show that

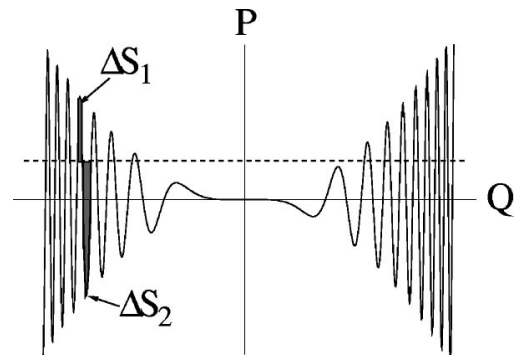

FIG. 2. Areas in phase space important to momentum wave function associated with the manifold $P(Q)=p$ $-(3 / 2) \hbar \alpha \lambda Q^{2} \sin \left(\alpha Q^{3}\right), \lambda=0.5$. Primitive semiclassical approximation fails since both areas $\Delta S_{1}$ and $\Delta S_{2}$ can be smaller than $\hbar$.

intricate manifolds can sometimes be replaced by much simpler ones, which may themselves be evaluated semiclassically in any basis, using a much simplified uniformization if required.

\section{REPLACEMENT MANIFOLDS}

We now introduce an example of such a problematic Lagrangian manifold, defined by a position-momentum relation

$$
P(Q)=p-\frac{3}{2} \hbar \lambda \alpha Q^{2} \sin \alpha Q^{3}
$$

(the use of capital letters will become clear in Sec. IV). This model was introduced in Ref. [4] but was not explored there. The function (11) has oscillations of increasing amplitude and frequency which, however, have the same area $\int P(Q) d Q=\lambda \hbar$ between successive zeros (see Fig. 2, small value of $\lambda$ corresponds to small area of loops). This is also a characteristic property of homoclinic oscillations near a periodic orbit. The momentum $P(Q)$ spans an ever larger range as $Q$ increases, but we now show that for $\lambda<1$ the manifold's semiclassical behavior can be understood by replacing it with three smooth manifolds. The rules for replacement are simple to derive.

The action function $S(Q)$ is the integral of $P(Q)$,

$$
S(Q)=\int_{0}^{Q} P\left(Q^{\prime}\right) d Q^{\prime}=p Q+\frac{1}{2} \hbar \lambda\left(\cos \alpha Q^{3}-1\right) .
$$

The "wave function"

$$
\psi(Q)=A(Q) e^{i S(Q) / \hbar}
$$

can be approximated by

$$
\begin{gathered}
\psi(Q) \approx A(Q) e^{-i \lambda / 2} e^{i p Q / \hbar}\left(1+i \frac{\lambda}{2} \cos \left(\alpha Q^{3}\right)\right) \\
\psi(Q) \approx A(Q) e^{-i \lambda / 2} e^{i p Q / \hbar}\left(1+i \frac{\lambda}{4} e^{i \alpha Q^{3}}+i \frac{\lambda}{4} e^{-i \alpha Q^{3}}\right)
\end{gathered}
$$

if $\lambda<1$. For small $\lambda$ this expression is nearly identical with Eq. (13) with the original action (12), because $\cos \left(\alpha Q^{3}\right)$ is 


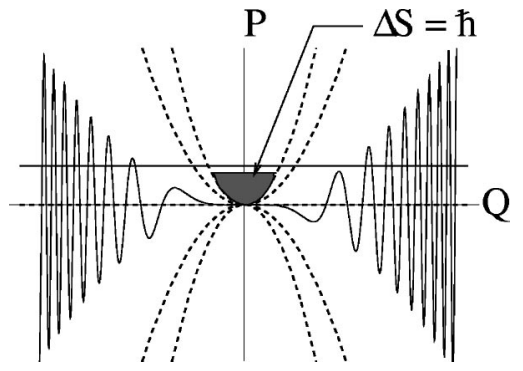

FIG. 3. Original (solid line) and replacement manifolds (dashed lines) for $P(Q)=p-(3 / 2) \hbar \alpha \lambda Q^{2} \sin \left(\alpha Q^{3}\right), \lambda=0.5$.

bounded by \pm 1 . However, semiclassically it has the interpretation of the sum of three smooth classical manifolds, i.e., $P=p, P=p+3 \hbar \alpha Q^{2}$, and $P=p-3 \hbar \alpha Q^{2}$. They have the weights $e^{-i \lambda / 2} A(Q), i(\lambda / 4) e^{-i \lambda / 2} A(Q), i(\lambda / 4) e^{-i \lambda / 2} A(Q)$, respectively. The situation is depicted in Fig. 3.

Almost every discussion of the relation of classical and quantum mechanics for chaotic systems alludes to quantum smoothing, but here we have seen explicitly one way this smoothing comes about. For all reasonable purposes the three smooth classical manifolds accurately replace the rapid oscillations of the original manifold. Note, too, that depending on the parameters the outlying manifolds $P=p$ $+3 \hbar \alpha Q^{2}$ and $P=p-3 \hbar \alpha Q^{2}$ lie far beyond the limits of the original distributions. We shall discuss interesting consequences of this in Sec. IV.

So far, we have considered the wave function only in position representation where caustics are absent and the semiclassical form (13) was accurate. The situation deteriorates drastically in momentum space. In order to find the wave function in momentum representation, we have to sum over all contributions from intersections of a horizontal line (corresponding to a momentum eigenstate) with the oscillating manifold (11). When $\lambda<1$, the adjacent intersections will be separated by a phase $\Delta S / \hbar$ that is smaller than 1 for any classically allowed momentum, and therefore the standard semiclassical approximation will break down for all classical momenta. The usual Airy-type uniformization methods of Sec. II (also see $[5,6]$ ) do not serve under these circumstances because the manifold is dense with caustics (see the filled-in areas $\Delta S_{1}$ and $\Delta S_{2}$ in Fig. 2). Apparently, we have no other choice but to part with the semiclassical method and use a numerical Fourier transform (IVR) over the position wave function to obtain $\phi(P)$. However, using the replacement manifolds (RMs) is a much simpler and more intuitive approach which can rescue the situation.

To find the momentum wave function $\phi(P)$ associated with the original manifold we add (with appropriate weights) wave functions $\phi_{n}(P)$ corresponding to the three RMs. Each of these partial wave functions is equal to a Fourier transform of a corresponding position wave function $\psi_{n}(Q)$,

$$
\phi_{n}(P)=\frac{1}{\sqrt{2 \pi \hbar}} \int d Q \psi_{n}(Q) e^{-i P Q / \hbar}
$$

Except for a small region close to $p$ (highlighted in Fig. 3), this integral can be evaluated by the SP method. In other words, although the semiclassical approximation completely fails for the original manifold, it works almost everywhere for the replacement manifolds. Moreover, as we show in the following section, the standard Airy uniformization procedure of Sec. II can be exploited to correct the inaccuracy in a narrow region near $p$. We will see that the momentum wave function found by applying SP approximation to RMs is in excellent agreement with numerical solution. We emphasize that the RMs do not yield the original, badly behaved semiclassical result, but rather something much more accurate and much simpler.

\section{UNIFORMIZATION WITH REPLACEMENT MANIFOLDS IN A GENERALIZED MODEL}

In order to capture other properties of homoclinic oscillations in our model, we consider a generalized manifold

$$
P(Q)=p-\frac{1}{2} \hbar \lambda \frac{d \xi(Q)}{d Q} \sin \xi(Q),
$$

in which $\alpha Q^{3}$ from Eq. (11) is replaced by a generic smooth function $\xi(Q)$. The presence of the derivative $d \xi(Q) / d Q$ ensures equal loop areas between successive zeros of $\xi(Q)$. Example of a manifold with $\xi(Q)=\alpha \log (Q / a)$ is displayed in Fig. 5. Manifold (17) can be obtained from a horizontal manifold representing a momentum state $|p\rangle$ by a canonical transformation of coordinates [7]

$$
Q=q, \quad P=p-\frac{1}{2} \hbar \lambda \frac{d \xi}{d q} \sin \xi(q),
$$

generated by a function

$$
F_{3}(p, Q)=-p Q-\frac{1}{2} \hbar \lambda \cos \xi(Q),
$$

We find the uniform semiclassical transformation element $\langle P \mid p\rangle$, and thereby fix the amplitude prefactor to be $A(Q)$ $=(2 \pi \hbar)^{-1 / 2}$; this, however, retains all the problematic features of the manifold. We solve the problem to all orders in $\lambda$ by replacing the original manifold with an infinite series of RMs. First we note that the semiclassical transformation element

$$
\langle Q \mid p\rangle_{s c}=\frac{1}{\sqrt{2 \pi \hbar}} e^{-i F_{3}(p, Q) / \hbar}
$$

is fairly accurate since there are no caustics in this representation (to each final $Q$ corresponds a single initial $q$ ). The momentum-representation element is obtained by a Fourier transform

$$
\begin{aligned}
\langle P \mid p\rangle & \simeq \frac{1}{\sqrt{2 \pi \hbar}} \int d Q e^{-i P Q / \hbar}\langle Q \mid p\rangle_{s c} \\
& =\frac{1}{2 \pi \hbar} \int d Q \exp \left\{\frac{i}{\hbar}\left[-P Q-F_{3}(p, Q)\right]\right\} .
\end{aligned}
$$


If evaluated by the SP method, the integral yields a very poor semiclassical result since there exist many coalescing SP points. An accurate uniform answer is obtained by evaluating integral (21) exactly. The accuracy is usually further improved by changing the integration variable from $Q$ to $q$ (in our case both forms are equivalent since $Q=q$ ). This uniform version of $\langle P \mid p\rangle$ is in IVR form [2,3], but can best be evaluated by writing $\langle Q \mid p\rangle$ as a sum over RMs. Recognizing that the factor $e^{i(\lambda / 2) \cos \xi(Q)}$ in $\langle Q \mid p\rangle_{s c}$ $=(2 \pi \hbar)^{-1 / 2} \exp [i p Q / \hbar+i(\lambda / 2) \cos \xi(Q)]$ is a generating function for Bessel functions, we can extend the sum from Sec. III beyond the first order in $\lambda$. In fact we obtain an infinite sum convergent for any $\lambda$,

$$
\begin{aligned}
\langle Q \mid p\rangle & =\frac{1}{\sqrt{2 \pi \hbar}} e^{i p Q / \hbar} \sum_{n=-\infty}^{\infty} J_{n}\left(\frac{\lambda}{2}\right) i^{n} e^{i n \xi(Q)} \\
& =\sum_{n=-\infty}^{\infty} J_{n}\left(\frac{\lambda}{2}\right) i^{n}\langle Q \mid p\rangle_{n},
\end{aligned}
$$

where $\langle Q \mid p\rangle_{n}=(2 \pi \hbar)^{-1 / 2} \exp \{i[p Q+n \hbar \xi(Q)] / \hbar\}$. We can rewrite Eq. (21) as

$$
\begin{aligned}
\langle P \mid p\rangle & =\sum_{n=-\infty}^{\infty} J_{n}\left(\frac{\lambda}{2}\right) \frac{i^{n}}{\sqrt{2 \pi \hbar}} \int d Q e^{-i P Q / \hbar}\langle Q \mid p\rangle_{n} \\
& =\sum_{n=-\infty}^{\infty} J_{n}\left(\frac{\lambda}{2}\right) i^{n}\langle P \mid p\rangle_{n},
\end{aligned}
$$

where

$$
\langle P \mid p\rangle_{n}=\frac{1}{2 \pi \hbar} \int d Q \exp \left\{\frac{i}{\hbar}[(p-P) Q+n \hbar \xi(Q)]\right\}
$$

may be identified semiclassically as a transformation element corresponding to the $n$th RM

$$
P_{n}(Q)=p+n \hbar \frac{d \xi(Q)}{d Q} .
$$

This manifold for most functions $\xi(Q)$ of interest contains no caustics, and consequently $\langle P \mid p\rangle_{n}$ allows evaluation by the SP method. As promised, we have expressed the uniform version of $\langle P \mid p\rangle$ as a weighted sum over semiclassical replacement manifolds,

$$
\langle P \mid p\rangle_{\text {unif }}=\sum_{n=-\infty}^{\infty} J_{n}\left(\frac{\lambda}{2}\right) i^{n}\langle P \mid p\rangle_{n, s c} .
$$

If applied to a well-behaved (i.e., decaying fast enough at $\pm \infty$ ) function of $p$, the resulting sum converges for any $\lambda$. The replacement-manifold method is therefore not restricted to the regime where loop areas are smaller than $\hbar$. It also works in the strongly chaotic regime where $\lambda>1$ (and where the standard semiclassical approximation holds) if all manifolds up to $|n| \simeq \lambda$ are included in the sum. This follows since $J_{n}(\lambda / 2)$ considered as a function of $n$ decays exponen- tially fast for $|n|>\lambda / 2$. In other words, we only need to include several RMs beyond those intersecting the original manifold. Physically, the manifolds outside of the range of the original one lie in a classically forbidden region in which the wave function is exponentially suppressed.

In the opposite case $(\lambda<1)$ which interests us the most, considering RMs up to $|n|=1$ will suffice while the "primitive" semiclassical approximation fails completely even for $P=p$. In this regime $\lambda$ can be thought of as a parameter defining the strength of a perturbation which causes the manifold to oscillate around a simple manifold that describes the system if perturbation is absent.

To complete our solution for a specific function $\xi(Q)$, we must evaluate $\langle P \mid p\rangle_{n}$. For $n=0$, we cannot use the SP approximation (because the action is linear), but integral (24) is trivial and we obtain in general

$$
\langle P \mid p\rangle_{0}=\delta(P-p) .
$$

For $n \neq 0$, the SP method applied to Eq. (24) yields a result in the form of a sum over SP points $Q_{s p}$,

$$
\begin{aligned}
\langle P \mid p\rangle_{n, s c}= & \frac{1}{\sqrt{2 \pi \hbar}} \sum_{Q=Q_{s p}}\left|\frac{\partial^{2} f_{n}}{\partial Q^{2}}\right|^{-1 / 2} \\
& \times \exp \left[i\left(\frac{f_{n}}{\hbar}+\frac{\pi}{4} \operatorname{sgn} \frac{\partial^{2} f_{n}}{\partial Q^{2}}\right)\right],
\end{aligned}
$$

where $f_{n}(Q, P, p)=(p-P) Q+n \hbar \xi(Q)$ and $\partial f_{n} / \partial Q=0$ for $Q=Q_{s p}$. For manifold (11), each RM $P_{n}(Q)=p$ $+3 n \hbar \alpha Q^{2}$ has two SP points

$$
Q_{S P}= \pm\left(\frac{P-p}{3 n \hbar \alpha}\right)^{1 / 2}
$$

whose contributions add to give

$$
\begin{aligned}
\langle P \mid p\rangle_{n, s c}= & \pi^{-1 / 2}\left[3 n \hbar^{3} \alpha(P-p)\right]^{-1 / 4} \\
& \times \cos \left[\frac{2}{(n \alpha)^{1 / 2}}\left(\frac{P-p}{3 \hbar}\right)^{3 / 2}-\frac{\pi}{4}\right] .
\end{aligned}
$$

RMs still contain one caustic at $P=p$, which can be easily uniformized by evaluating integral (24) exactly, as in Sec. II [8],

$$
\langle P \mid p\rangle_{n, \text { unif }}=\frac{1}{(3|n| \alpha)^{1 / 3} \hbar} \operatorname{Ai}\left(-\frac{(\operatorname{sgn} n)(P-p)}{(3|n| \alpha)^{1 / 3} \hbar}\right) .
$$

Having found $\langle P \mid p\rangle_{n}$, we can calculate $\langle P \mid p\rangle$ from Eq. (23) to all orders in $\lambda .\langle P \mid p\rangle_{1, s c}$ and $\langle P \mid p\rangle_{1, \text { unif }}$ agree very well except for a small region near $p$ (similarly as in Fig. 1). Figure 4 demonstrates the excellent agreement of the RM method with the direct numerical computation of $\langle P \mid p\rangle$ using fast Fourier transform (IVR).

Here the replacement-manifold approach should be compared to the semiclassical perturbation (SCP) approximation of Miller and Smith $[9,10]$ who used perturbative classical dynamics to calculate the semiclassical $S$ matrix. They first 


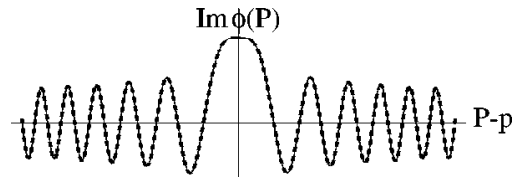

FIG. 4. Momentum wave function: comparison of numerical IVR (solid line) with the RM sum up to $|n|=1[\mathrm{O}(\lambda)$, dashed line $]$ for the original manifold with $\xi(Q)=\alpha Q^{3}, \lambda=0.5$, and $\alpha=1$.

applied this method to the collinear collision of an atom with a diatomic molecule [9]. The starting point is the initial value representation of the classical scattering matrix,

$$
\begin{aligned}
S_{n_{f}, n_{i}}= & \frac{1}{2 \pi} \int_{0}^{2 \pi} d q_{i}\left[\frac{\partial q_{f}\left(q_{i}, n_{i}\right)}{\partial q_{i}}\right]^{1 / 2} \\
& \times \exp \left(i\left\{\Phi\left(q_{i}, n_{i}\right)+q_{f}\left(q_{i}, n_{i}\right)\left[n_{f}\left(q_{i}, n_{i}\right)-n_{f}\right]\right\}\right),
\end{aligned}
$$

where $\hbar=1, n_{i}, n_{f}, q_{i}, q_{f}$ denote, respectively, the initial and final values of the action and angle variables describing the internal degree of freedom, and $\Phi$ is the action integral. For details, see Refs. [2] and [9]. If the classical quantities listed above are calculated using the first-order perturbation dynamics, the $S$ matrix takes the form

$$
S_{n_{f}, n_{i}}=\frac{e^{i \Phi_{0}}}{2 \pi} \int_{0}^{2 \pi} d q_{i} \exp \left\{-i\left[\left(n_{f}-n_{i}\right) q_{i}+A\left(q_{i}, n_{i}\right)\right]\right\}
$$

where $\Phi_{0}$ is twice the phase shift for the unperturbed potential and $A\left(q_{i}, n_{i}\right)$ is the time integral of the perturbation potential along the unperturbed trajectory [9]. For various physical systems (such as ion-dipole collisions [9] or atomsurface scattering [10]), $A\left(q_{i}, n_{i}\right)$ has a sinusoidal dependence on $q_{i}$ as long as perturbation remains small. In this regime we expect the SCP and RM approximations to give the same answer. We shall verify that for the scattering by a corrugated wall in Sec. VII. The SCP approximation of Miller and Smith presently appears to be more widely applicable since it does not require any special property of the manifold. On the other hand, the advantage of the RM method lies in the fact that it is not subjected to the smallness of perturbation. Provided that the full action falls into one of the classes discussed above, we can apply the RM method without approximating classical dynamics.

\section{MODEL OF HOMOCLINIC OSCILLATIONS}

Homoclinic oscillations in chaotic systems have another characteristic property: the amplitude of oscillations (i.e., "height" of loops) increases exponentially as we approach an unstable periodic orbit (see Fig. 5).

To keep the area of loops constant, the width of loops has to decrease accordingly. Because systems in nature are usually bounded, the exponentially growing loops must eventually twist in a complicated way to fit into available phase space. For the sake of simplicity, we consider a model that satisfies the requirement of exponential growth, but is unbounded. Such a model can be found among the generalized

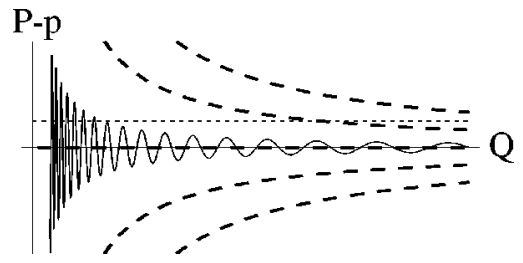

FIG. 5. Original (solid line) and replacement manifolds (dashed lines) for $\xi(Q)=\alpha \log (Q / a), \lambda=0.5$.

manifolds of the preceding section. Specifically, we want to find a manifold whose homoclinic points (i.e., intersections with line $P=p$ in our case) form a geometric series,

$$
Q_{n}=a e^{n \lambda_{+}},
$$

where $\lambda_{+}$is the Lyapunov exponent. In other words, we are looking for a function $\xi(Q)$ such that $\xi\left(Q_{n}\right)=2 \pi n$. The logarithmic function comes to mind first since $\log \left(Q_{n} / a\right)$ $=n \lambda_{+}$. With the correct prefactor $\alpha=2 \pi / \lambda_{+}$, we find the desired function

$$
\xi(Q)=\alpha \log \frac{Q}{a}
$$

because $\xi\left(Q_{n}\right)=\alpha \log \left(Q_{n} / a\right)=\left(2 \pi / \lambda_{+}\right) n \lambda_{+}=2 \pi n$. Substituting $\xi(Q)$ from Eq. (32) into the general form (17), we obtain a manifold described by

$$
P=p-\frac{1}{2} \frac{\hbar \lambda \alpha}{Q} \cos \left(\alpha \log \frac{Q}{a}\right)
$$

and displayed in Fig. 5. Replacement manifolds (19) become

$$
P_{n}(Q)=p+\frac{n \hbar \alpha}{Q}
$$

In the present case, function $f_{n}$ from Eq. (27) becomes

$$
f_{n}(Q, P, p)=(p-P) Q+n \hbar \alpha \log \frac{Q}{a},
$$

and its single SP point,

$$
Q_{s p}=\frac{n \hbar \alpha}{P-p} .
$$

For $P>p$, only manifolds with $n>0$ contribute, and for $P$ $<p$ only $n<0$ is allowed. Defining $j=|n|$, for $P \neq p$ we replace the sum over $n$ by sum over all positive $j$. In both cases, the $j$ th manifold gives a semiclassical contribution

$$
\begin{aligned}
\langle P \mid p\rangle_{j, s c}= & \left(\frac{j \alpha}{2 \pi}\right)^{1 / 2} \frac{1}{|P-p|} \exp \{i \operatorname{sgn}(P-p) \\
& \left.\times\left[j \alpha \log \frac{j \hbar \alpha}{a e|P-p|}-\frac{\pi}{4}\right]\right\}
\end{aligned}
$$

with $j=|n|>0$. In this case caustics are missing because there is a single $\mathrm{SP}$ point $Q_{s p}$. We have a finite integration 


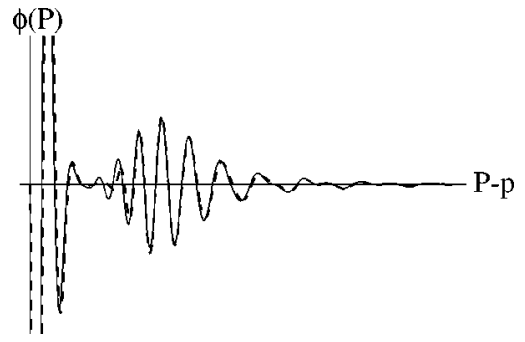

FIG. 6. Momentum wave function: comparison of numerical IVR (solid line) with the RM sum up to $j=1[O(\lambda)$, dashed line $]$ for the original manifold with $\xi(Q)=\alpha \log (Q / a), \lambda=0.5$, and $\alpha$ $\approx 33$.

limit at zero, but it is separated from $Q_{s p}$ by an infinite action (equal to the area delimited by lines $Q=0, P=p$, and the $n$th RM, see Fig. 5). In fact, it can be shown that further terms in asymptotic expansion of $\langle P \mid p\rangle_{j, s c}$ have the same dependence on $\hbar$ and $P-p$ as Eq. (36), and only differ in their dependence on $j \alpha$. Luckily, these claims can be easily verified by evaluating integral (24) analytically, which is done in the Appendix.

In Fig. 6 the replacement-manifold expansion of $\langle P \mid p\rangle$ is applied to an initial Gaussian wave packet centered around $q_{0}, p_{0}$, namely,

$$
\phi^{i}(p)=\left(\frac{\sigma^{2}}{\pi \hbar^{2}}\right)^{1 / 4} \exp \left[\frac{i}{\hbar}\left(p_{0}-p\right) q_{0}-\frac{\sigma^{2}\left(p-p_{0}\right)^{2}}{2 \hbar^{2}}\right] .
$$

The final position wave function is

$$
\psi_{s c}^{f}(Q)=\left(\pi \sigma^{2}\right)^{-1 / 4} \exp \left[-\frac{i}{\hbar} F_{3}\left(p_{0}, Q\right)-\frac{\left(Q-q_{0}\right)^{2}}{2 \sigma^{2}}\right]
$$

and the uniform momentum wave function,

$$
\begin{aligned}
\phi_{\text {unif }}^{f}(P)= & J_{0}\left(\frac{\lambda}{2}\right) \phi^{i}(P)+\frac{(2 \pi \hbar)^{1 / 2}}{\left(\pi \sigma^{2}\right)^{1 / 4}} \\
& \times \sum_{j=1}^{\infty} J_{j}\left(\frac{\lambda}{2}\right) i^{j}\left\langle P \mid p_{0}\right\rangle_{j, s c} \exp \left[-\frac{\left(Q_{s p, j}-q_{0}\right)^{2}}{2 \sigma^{2}}\right]
\end{aligned}
$$

with $Q_{s p, j}=j \hbar \alpha /\left|P-p_{0}\right|$ and $\left\langle P \mid p_{0}\right\rangle_{j, s c}$ given by Eq. (36).

The large oscillation near $P=p_{0}$ in Fig. 6 corresponds to the zeroth RM contribution $\phi^{i}(P)$; the smaller wavelet to the right corresponds to the remaining RMs. The figure confirms the excellent accord between the RM method and the numerical IVR evaluation by fast Fourier transform (FFT) [11]. We have thus succeeded in uniformizing something with many of the properties of homoclinic oscillations near an unstable periodic orbit using replacement-manifold "technology."

\section{COMPARISON WITH THE STATIONARY-PHASE METHOD}

It is instructive to check explicitly if the expansion in terms of RMs agrees with the semiclassical method applied to the original manifold when areas of loops are larger than $\hbar$ $(\lambda>1)$. Let us choose an analytically solvable example with $\xi(Q)=\alpha Q$. The corresponding manifold

$$
P(Q)=p-\frac{1}{2} \hbar \lambda \alpha \sin \alpha Q
$$

has another advantage compared to manifolds in Figs. 3 and 5. Unlike those, for $\lambda \gg 1$ and small enough $P-p$, manifold (38) has all caustics in a safe distance. The RMs are horizontal lines $P_{n}=p+n \hbar \alpha$, independent of $Q$ and corresponding transformation elements can be evaluated exactly as $\langle P \mid p\rangle_{n}$ $=\delta(P-p-n \hbar \alpha)$. Using Eq. (23), the uniform expression for $\langle P \mid p\rangle$ is

$$
\langle P \mid p\rangle_{\text {unif }}=\sum_{n=-\infty}^{\infty} J_{n}\left(\frac{\lambda}{2}\right) i^{n} \delta(P-p-n \hbar \alpha),
$$

so the wave function is determined by its Fourier coefficients $a_{n}(\lambda)=J_{n}(\lambda / 2) i^{n}$, which is natural since manifold (38) is exactly periodic. Moreover, each RM contributes only to a single momentum $\left(P_{n}\right)$. Put differently, we have calculated the wave function for any specific momentum to all orders in $\lambda$. Now let us find the semiclassical form of $\langle P \mid p\rangle$. For a given momentum $P$ there is an infinite number of SP points $Q_{S P}$ which occur in pairs $\left(X_{n}, Y_{n}\right)$,

$$
X_{n}=X_{0}+\frac{2 \pi}{\alpha} n, \quad Y_{n}=\frac{\pi}{\alpha}-X_{0}+\frac{2 \pi}{\alpha} n,
$$

where $X_{0}=-(1 / \alpha) \arcsin [2(P-p) / \lambda \hbar \alpha]$. Analogously to Eq. (27), the semiclassical transformation element for manifold (38) is

$$
\begin{aligned}
\langle P \mid p\rangle_{s c}= & \frac{1}{\sqrt{2 \pi \hbar}} \sum_{n=-\infty}^{\infty}\left|\frac{\partial^{2} S}{\partial Q^{2}}\right|_{Q=X_{n}}^{-1 / 2}\left\{\exp \left[\frac{i}{\hbar} S\left(X_{n}\right)-i \frac{\pi}{4}\right]\right. \\
& \left.+\exp \left[\frac{i}{\hbar} S\left(Y_{n}\right)+i \frac{\pi}{4}\right]\right\}
\end{aligned}
$$

with

$$
S(Q, P, p)=-P Q-F_{3}(p, Q)=(p-P) Q+\frac{1}{2} \hbar \lambda \cos \alpha Q .
$$

Using Eqs. (40) and (41) and defining $r=(P-p) / \hbar \alpha$, we get

$$
\langle P \mid p\rangle_{s c}=\frac{1}{\hbar \alpha} F(\lambda, r) \sum_{n=-\infty}^{\infty} e^{2 \pi i n r},
$$

where 
TABLE I. Comparison of numerical, RM, and SP evaluations of coefficients $a_{n}$ of the momentum wave function for $\xi(Q)=\alpha Q, \lambda$ $=10$.

\begin{tabular}{lcccccc}
\hline \hline$n$ & 0 & 1 & 2 & 3 & 4 & 5 \\
\hline$\left|a_{n}\right|$ (num.) & 0.1776 & 0.3276 & 0.0466 & 0.3648 & 0.3912 & 0.2611 \\
$\left|a_{n}\right|(\mathrm{RM})$ & 0.1776 & 0.3276 & 0.0466 & 0.3648 & 0.3912 & 0.2611 \\
$\left|a_{n}\right|$ (SP) & 0.1704 & 0.3324 & 0.0343 & 0.3622 & 0.4312 & $\infty$ \\
\hline \hline
\end{tabular}

$$
\begin{aligned}
F(\lambda, r)= & \sqrt{\frac{2}{\pi}} e^{-i r \pi / 2} \\
& \times \frac{\cos \left\{r\left(\frac{\pi}{2}+\arcsin \frac{2 r}{\lambda}\right)+\left[\left(\frac{\lambda}{2}\right)^{2}-r^{2}\right]^{1 / 2}-\frac{\pi}{4}\right\}}{\left[\left(\frac{\lambda}{2}\right)^{2}-r^{2}\right]^{1 / 4}} .
\end{aligned}
$$

Employing the Poisson summation formula

$$
\sum_{n=-\infty}^{\infty} e^{2 \pi i n r}=\sum_{m=-\infty}^{\infty} \delta(m-r)
$$

and reverting to $P=p+r \hbar \alpha$, we obtain

$$
\begin{aligned}
\langle P \mid p\rangle_{s c} & =\frac{1}{\hbar \alpha} F(\lambda, r) \sum_{n=-\infty}^{\infty} \delta(n-r) \\
& =\frac{1}{\hbar \alpha} \sum_{n=-\infty}^{\infty} F(\lambda, n) \delta(n-r) \\
& =\sum_{n=-\infty}^{\infty} F(\lambda, n) \delta(P-p-n \hbar \alpha) .
\end{aligned}
$$

Comparing Eq. (44) with Eq. (39), we see that the semiclassical and uniform versions of $\langle P \mid p\rangle$ will be asymptotically equal if $F(\lambda, n) \sim J_{n}(\lambda / 2) i^{n}$ for large $\lambda$. That is indeed true since for $\lambda \gg n^{2}$,

$$
F(\lambda, n) \sim\left(\frac{2}{\pi \frac{\lambda}{2}}\right)^{1 / 2} e^{i n \pi / 2} \cos \left(\frac{\lambda}{2}-\frac{n \pi}{2}-\frac{\pi}{4}\right) \sim J_{n}\left(\frac{\lambda}{2}\right) i^{n} .
$$

Table I shows that for $\lambda=10$ semiclassical and RM values of $a_{n}$ differ by less than 0.02 up to $n=3$. In the opposite limit, for $\lambda<1$, even $a_{0}$ evaluated semiclassically is completely off.

\section{SCATTERING FROM A CORRUGATED WALL}

Let us apply the replacement-manifold method to a physical problem generating loop structure in phase space. Scattering of a plane wave by a corrugated wall has exactly such a property. This system has been used to model elastic scattering of atoms by solid surfaces (for review see [12]).

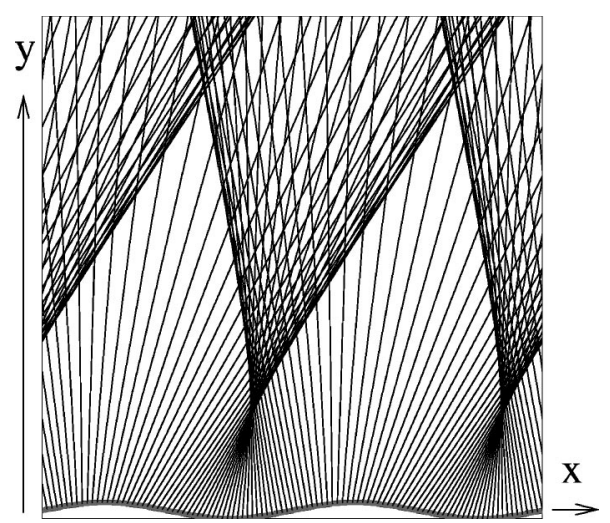

FIG. 7. Ray picture for a two-dimensional scattering from a corrugated wall. Only reflected rays are shown.

Consider a plane divided into two parts by a periodically curved boundary consisting of the set of points with coordinates related by

$$
\tilde{y}(x)=\frac{\varepsilon}{\beta} \sin \beta x,
$$

where $\beta$ gives the spatial frequency of corrugation and $\varepsilon$ the maximum slope $d \tilde{y} / d x$ of the wall. A plane wave with momentum $\hbar \mathbf{k}_{1}$ is incident from the upper half-plane at an angle $\alpha_{1}$ from the $y$ axis, so the incident wave vector is $\mathbf{k}_{1}$ $=k\left(\sin \alpha_{1},-\cos \alpha_{1}\right)$. Classically, the wave reflects specularly from the curved boundary. The reflected rays are shown in Fig. 7.

Notice also the clearly visible caustics, emanating in pairs from cusp singularities. The Poincare surface of section in Fig. 8 displays the dependence of momentum component $p_{2 x}$ of reflected rays on coordinate $x$ at a given distance $y_{2}$ from $x$ axis. We can see familiar loops with constant area and predict the failure of a semiclassical approximation when this area gets smaller than $\hbar$. The figure implicitly assumes that classically only a single scattering event takes place before a ray leaves the wall permanently. If corrugation is deep enough $\left(\varepsilon>\varepsilon_{\max }\right.$, where $\left.\varepsilon_{\max }<1\right)$, multiple scattering will occur for any incident angle $\alpha_{1}$. The larger $\varepsilon$ gets (within the range $0<\varepsilon<\varepsilon_{\max }$ ), the smaller the maximum allowed incident angle $\alpha_{1}$. Let us therefore consider a wall with very shallow corrugation $(\varepsilon=0.2)$ and a small angle of incidence $\left(\alpha_{1}=0.2\right.$ ), a situation which classically allows only a single reflection. We also choose the incident-wave length $\lambda$ $=2 \pi / k$ to be 0.3 times the period of corrugation. The surprising result we shall obtain below is that although there

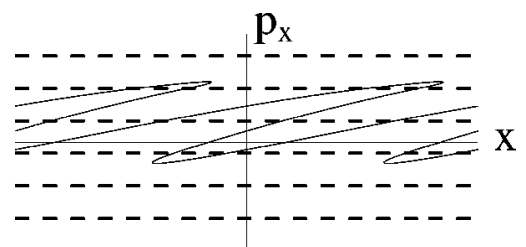

FIG. 8. Original (solid line) and replacement manifolds (dashed lines) on the Poincaré surface of section at $y_{2}=4 \pi / \beta$. 


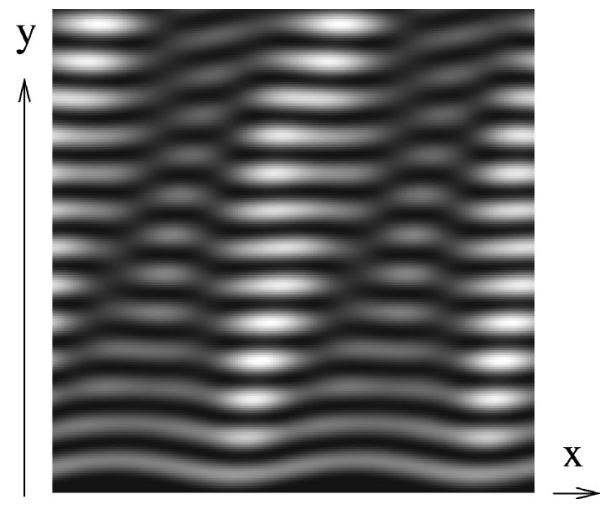

FIG. 9. Probability density plot for the exact quantum solution. Plot of the RM solution is indistinguishable to the eye. For detailed comparison, see Fig. 11.

exist seven real Bragg peaks in the scattered wave, the semiclassics break down while the single-scattering approximation using replacement manifolds works with excellent accuracy. Before applying the RM method, let us briefly mention what exact and semiclassical solutions are used for comparison.

The most direct, Rayleigh approach $[13,14]$ to find exact solution relies on expanding the scattered wave in Fourier modes

$$
\psi_{\text {scat }}(\mathbf{r})=\frac{1}{2 \pi} \sum_{n=-\infty}^{\infty} a_{n} e^{i \mathbf{k}_{2, n} \cdot \mathbf{r}}
$$

and finding the coefficients $a_{n}$ so that the total wave function vanishes along the boundary. Garcia and Cabrera [15] have thoroughly compared merits of this and various other methods. The main issue is the solution's convergence, which improves with decreasing corrugation parameter $\epsilon$. However, we can safely use the Rayleigh method since it has been shown to converge for $\epsilon<0.448$ [16] and this regime overlaps with the small-loop limit in which we are interested. The resulting probability density is shown in Fig. 9.

The simple semiclassical wave function may be evaluated by tracing individual rays and employing the Van Vleck propagator [17]. The action must be adjusted by correct Maslov indices, corresponding to reflection from a hard wall and to passage through caustics [18]. A probability density

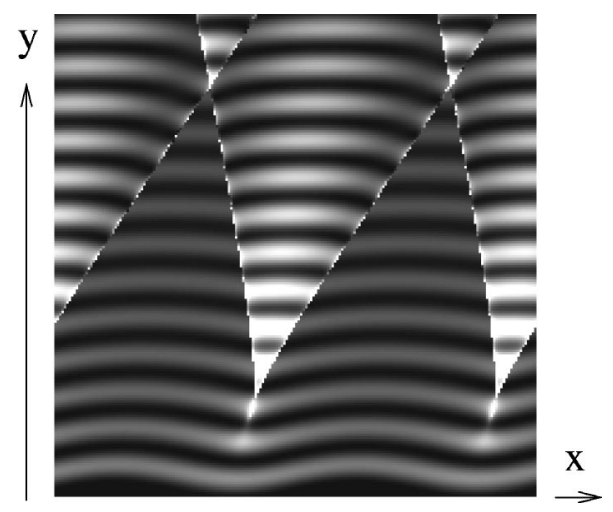

FIG. 10. Probability density plot for the semiclassical solution. plot of the semiclassical wave function is shown in Fig. 10. Note the caustics, which clearly separate regions with one, three, and five contributions to the scattered wave. Between caustics, solution looks qualitatively the same as the exact quantum analogue in Fig. 9. By looking at a surface of section at $y_{2}=4 \pi / \beta$ (corresponding to the top edge of Fig. 10 where there are at least three contributions for any $x$ ) in Fig. 8 , we expect that semiclassical and quantum wave functions should disagree everywhere. This guess is confirmed in Fig. 11.

The application of replacement manifolds is facilitated if the whole scattering problem is formulated using an analog to the smooth-potential Lippmann-Schwinger equation. Invoking the Green's theorem, it can be shown that the total wave function satisfies

$$
\left|\psi_{\text {tot }}\right\rangle=\left|\psi_{\text {inc }}\right\rangle+\hat{G}_{0} \int_{\text {boundary }} d x|\mathbf{r}\rangle \hat{\mathbf{n}}(x) \cdot \nabla \psi_{\text {tot }}(\mathbf{r}),
$$

where $\left|\psi_{i n c}\right\rangle$ is the incident wave, $\hat{G}_{0}$ is the free-space Green's operator, and $\hat{\mathbf{n}}(x)$ is a normal unit vector at the boundary pointing into free space.

We find $\left|\psi_{t o t}\right\rangle$ in Eq. (48) by proceeding in four steps: first we canonically transform the incident wave function to a new coordinate system $\mathbf{r}^{\prime}$ in which the wall becomes straight. We solve the scattering problem in these coordinates, since there the semiclassical approach is exact. Then we transform back to the original coordinate system in which we propagate the wave using the free-space Green's function to obtain our final answer. The semiclassical approach is used for each separate step but their combination is evaluated exactly.

The wall becomes flat if we apply a canonical transformation

$$
x^{\prime}=x, \quad y^{\prime}=y-\frac{\varepsilon}{\beta} \sin \beta x
$$

generated by

$$
F_{3}\left(\mathbf{k}, \mathbf{r}^{\prime}\right)=-k_{x} x^{\prime}-k_{y}\left(y^{\prime}+\frac{\varepsilon}{\beta} \sin \beta x^{\prime}\right) .
$$

The incident wave function $\psi_{\text {inc }}\left(\mathbf{k}_{1}\right)$ can be transformed into new coordinates using

$$
\left\langle\mathbf{r}^{\prime} \mid \mathbf{k}\right\rangle_{s c}=\frac{1}{2 \pi} e^{-i F_{3}\left(\mathbf{k}, \mathbf{r}^{\prime}\right)} .
$$

In primed coordinates, the semiclassical solution of scattering is exact and the "angle of incidence" equals the "angle of reflection." Therefore $\hat{\mathbf{n}}^{\prime} \cdot \boldsymbol{\nabla} \psi_{t o t}\left(\mathbf{r}^{\prime}\right)$ may be replaced by $2 \hat{\mathbf{n}}^{\prime} \cdot \boldsymbol{\nabla} \psi_{\text {inc }}\left(\mathbf{r}^{\prime}\right)$ in Eq. (48). The nondiagonal part of the semiclassical scattering matrix becomes

$$
\hat{T}=\hat{S}-\hat{1}=2 \int d x^{\prime}\left|\mathbf{r}^{\prime}\right\rangle \hat{n}^{\prime} \cdot \nabla^{\prime}\left\langle\mathbf{r}^{\prime}\right| \|_{y^{\prime}=0},
$$


where $\hat{\mathbf{n}}^{\prime}=\hat{\mathbf{y}}^{\prime}$. Transformation back to the original coordinates is achieved by a complex conjugate of Eq. (50). The free-space propagation is accomplished by a semiclassical Green's function, exact in mixed representation

$$
\left\langle k_{2 x}, y_{2}\left|\hat{G}_{0}^{s c}\right| \mathbf{k}\right\rangle=\frac{\delta\left(k_{2 x}-k_{x}\right) e^{i k_{y} y_{2}}}{\sqrt{2 \pi}\left(k^{2}-k_{2}^{2}+i \epsilon\right)} .
$$

Combining all four pieces, we obtain an expression for the scattered wave on a surface of section,

$$
\begin{aligned}
\left\langle k_{2 x}, y_{2}\left|\hat{G}_{0}^{s c} \hat{T}\right| \mathbf{k}_{1}\right\rangle= & 2 \int d^{2} k \int d x^{\prime}\left\langle k_{2 x}, y_{2}\left|\hat{G}_{0}^{s c}\right| \mathbf{k}\right\rangle \\
& \times\left.\left\langle\mathbf{k} \mid \mathbf{r}^{\prime}\right\rangle \partial_{y^{\prime}}\left\langle\mathbf{r}^{\prime} \mid \mathbf{k}_{1}\right\rangle_{s c}\right|_{y^{\prime}=0} \\
= & \frac{2 i k_{1 y}}{(2 \pi)^{5 / 2}} \int d^{2} k \frac{\delta\left(k_{2 x}-k_{x}\right) e^{i k_{y} y_{2}}}{\sqrt{2 \pi}\left(k^{2}-k_{2}^{2}+i \epsilon\right)} \\
& \times \int d x^{\prime} \exp \left[i\left(k_{1 x}-k_{x}\right) x^{\prime}\right. \\
& \left.+i\left(k_{1 y}-k_{y}\right) \frac{\varepsilon}{\beta} \sin \beta x^{\prime}\right] .
\end{aligned}
$$

Before we proceed, it should be noted that the outcome of this four-step process, an integral representation of the scattered wave, can be viewed as a continuous superposition of waves emanating from sources along the boundary, with strength proportional to the normal derivative of the incident wave. It can be shown, in fact, that this process is equivalent to a generalized Kirchhoff diffraction method [19].

Returning to expression (53), we recognize that the exponent in integral over $x^{\prime}$ corresponds to a classical manifold

$$
k_{x}=k_{1 x}+\left(k_{1 y}-k_{y}\right) \varepsilon \cos \beta x^{\prime},
$$

which is "begging" to be replaced by partial manifolds $k_{x, n}=k_{1 x}+n \beta x^{\prime}$ with weights $J_{n}(\lambda)$ because

$$
|\lambda|=\left|k_{1 y}-k_{y}\right| \frac{\varepsilon}{\beta} \lesssim \frac{2 k \varepsilon}{\beta}<1
$$

for the classically allowed momenta. The integral over $x^{\prime}$ is then simple to evaluate and is equal to

$$
\begin{gathered}
\int d x^{\prime} \sum_{n=-\infty}^{\infty} J_{n}(\lambda) \exp \left[i\left(k_{1 x}+n \beta-k_{x}\right) x^{\prime}\right] \\
=2 \pi \sum_{n=-\infty}^{\infty} J_{n}(\lambda) \delta\left(k_{1 x}+n \beta-k_{x}\right) .
\end{gathered}
$$

Using this result and evaluating the trivial integral over $k_{x}$, expression (53) becomes

$$
\begin{aligned}
\left\langle k_{2 x}, y_{2}\left|\hat{G}_{0}^{s c} \hat{T}\right| \mathbf{k}_{1}\right\rangle= & \frac{2 i k_{1 y}}{(2 \pi)^{3 / 2}} \sum_{n=-\infty}^{\infty} \int d k_{y} \frac{e^{i k_{y} y_{2}}}{\sqrt{2 \pi}\left(k^{2}-k_{2}^{2}+i \epsilon\right)} \\
& \times J_{n}(\lambda) \delta\left(k_{2 x}-k_{2 x, n}\right),
\end{aligned}
$$

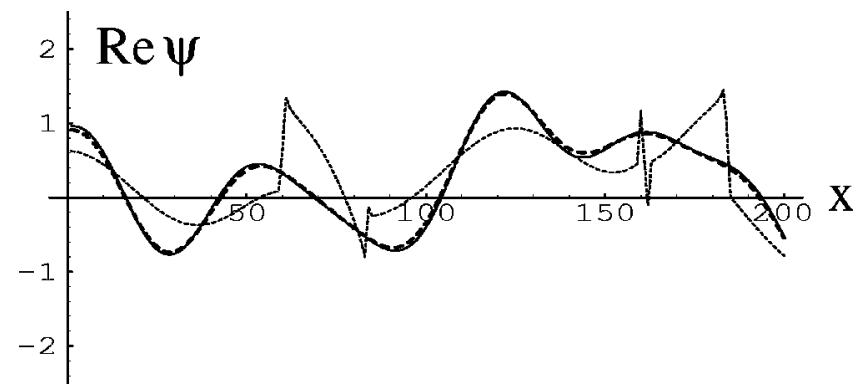

FIG. 11. Wave function on the surface of section at $y=4 \pi / \beta$. Comparison of the exact quantum (dashed line), RM (solid line), and semiclassical (dotted line) wave functions.

where $k_{2 x, n}=k_{1 x}+n \beta$. The integral over $k_{y}$ picks up a pole at $k_{y}=k_{2 y, n}=\sqrt{k^{2}-k_{2 x, n}^{2}}$ and the final answer is

$$
\begin{aligned}
& \left\langle k_{2 x}, y_{2}\left|\hat{G}_{0}^{s c} \hat{T}\right| \mathbf{k}_{1}\right\rangle \\
& =\frac{k_{1 y}}{\sqrt{2 \pi}} \sum_{n=-\infty}^{\infty} e^{i k_{2 y, n} y_{2}} \frac{J_{n}\left(\lambda_{n}\right) \delta\left(k_{2 x}-k_{2 x, n}\right)}{k_{2 y, n}},
\end{aligned}
$$

where $\lambda_{n}=\left(k_{1 y}-k_{2 y, n}\right)(\varepsilon / \beta)$. The uniform scattered wave is in the form (47) of a superposition of traveling and evanescent waves with wave vectors $\mathbf{k}_{2, n}$ and coefficients

$$
a_{n, u n i f}=\frac{k_{1 y}}{k_{2 y, n}} J_{n}\left(\lambda_{n}\right) \text {. }
$$

These turn out to be numerically very close to the coefficients of the exact solution, but here we have avoided having to solve a truncated linear system, which was necessary in Rayleigh or other exact methods [14].

A two-dimensional plot of probability density does not reveal any difference from the exact quantum result in Fig. 9 while an analogous semiclassical plot clearly shows caustics (see Fig. 10). Even if we look at the Poincare surface of section in a region with many classical caustics, the quantum and uniform solutions agree while the semiclassical solution fails miserably (see Fig. 11).

The uniform solution agrees with one found differently by Garibaldi et al. [20] and is somewhat more accurate than the same result without the $k_{1 y} / k_{2 y, n}$ prefactor obtained by Hubbard and Miller [10] by the semiclassical perturbation approximation. As Garibaldi et al. (who obtain three different solutions differing only by prefactors) point out, these prefactors "should not be taken too seriously." We agree: for traveling modes, they do not cause large errors, and since all these solutions neglect multiple scattering, we cannot expect high accuracy of the already small coefficients of evanescent modes.

Now we explain how semiclassical and single-scattering regimes differ. As mentioned above, the single-scattering approximation was guaranteed by the choice $\varepsilon \ll 1, \alpha \ll 1$. At first, it appears that the simple semiclassical approximation should be accurate for this regime of very shallow corrugation, since scattering from a flat wall has an exact semiclas- 
sical answer. The correct criterion, however, is based on the area of loops in phase space (see Fig. 8). In this case, for small $\varepsilon$ and $\alpha$, the height of the loops is equal to the momentum kick in the region of maximum slope, approximately $p \varepsilon$, and the width is half the period of corrugation, $\pi / \beta$. The loop area is smaller than $p \varepsilon(\pi / \beta)=\pi k d \hbar$ ( $d$ is the depth of corrugation) and has to be compared to $\hbar$. We arrive at a surprising result that the validity of the semiclassical approximation is unrelated to the periodicity of corrugation, but only depends on the product of the wave vector and corrugation depth. If this product is small, the semiclassical approximation breaks down. It works in the opposite case, although we might have to take into account classical trajectories corresponding to multiple scattering if $\epsilon \geqslant 1$.

It would be interesting to see in detail how uniform and semiclassical solutions get closer when both $k d \gg 1$ and $\epsilon$ $\ll 1$. While this could be shown explicitly, as in Sec. VI, by evaluating integrals in Eq. (53) by the SP method, Berry and Bodenschatz demonstrate the agreement for a similar problem of waves propagating normally from a sinusoidal wavefront [21] using the Talbot interference effect [22].

Finally, we remark that even in less fortunate situations when it is impossible to obtain analytical integrals for RMs, we can quite generally expect the replacement manifolds to be much smoother than the original one, enabling their simple evaluation by the SP method.

\section{CONCLUSION}

We have successfully uniformized several intimidating situations that can arise when repeated small areas or loops arise from enclosing classical manifolds using the replacement-manifold idea. This notion can rejuvenate physical intuition about the quantum wave function and also be a very convenient approach to an accurate result. Two physically motivated cases were considered (the homocliniclike oscillation and the corrugated wall) along with several other contrived cases to test the method. The present method should work in more complicated time-dependent problems if used with Miller's IVR applied to Van Vleck's semiclassical propagator. After replacement manifolds are identified, the perturbative expansion contributions to the wave function could be found by the SP approximation applied to these manifolds.

Future work along these lines includes the attempt to uniformize a full homoclinic tangle. It remains to be seen how convenient and generally applicable the replacement manifold idea is. However, it seems clear that we now have a method which simplifies many problems while reinstating an intuitive foundation.

\section{ACKNOWLEDGMENTS}

This research was supported by the National Science Foundation under Grant No. CHE9610501 and by the Institute for Theoretical Atomic and Molecular Physics. One of us (J. V.) would like to acknowledge helpful discussions with A. Mody and A. Barnett.

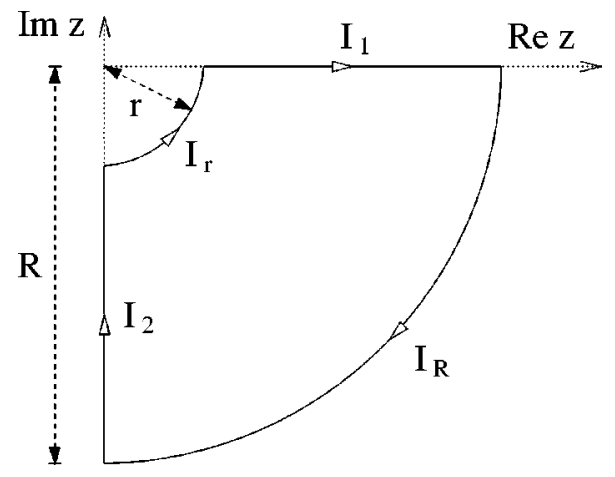

FIG. 12. Contour for evaluation of $\langle P \mid p\rangle_{n, \text { unif }}$ in the case $\xi(Q)=\alpha \log (Q / a)$.

\section{APPENDIX: EXACT EVALUATION OF A SINGLE REPLACEMENT MANIFOLD FOR THE MODEL OF HOMOCLINIC OSCILLATIONS}

The oscillatory integral (24) with $\xi(Q)=\alpha \log (Q / a)$ is made convergent by displacing momentum $P$ with an imaginary infinitesimal term $-i \epsilon, \epsilon>0$,

$$
\begin{aligned}
\langle P \mid p\rangle_{n, \text { unif }}= & \frac{1}{2 \pi \hbar} \int_{0}^{\infty} d Q \exp \left\{\frac{i}{\hbar}[-(P-i \epsilon-p) Q\right. \\
& \left.\left.+n \hbar \alpha \log \frac{Q}{a}\right]\right\} .
\end{aligned}
$$

In this form, the answer is found by rotating the contour about the origin of complex plane by $-(\pi / 2) \operatorname{sgn}(P-p)$.

Let us explicitly solve the case $P>p$. Figure 12 shows a contour in a complex plane enclosing a region with no singularities, and as a result the sum of integrals along appropriate parts of the contour is zero, $I_{1}+I_{R}+I_{2}+I_{r}=0$. In the limit $R \rightarrow \infty, r \rightarrow 0, I_{1}$ becomes our desired integral (A1) and both $I_{R}$ and $I_{r}$ vanish, implying $I_{1}=-I_{2}$. Contour for integral $I_{2}$ is the set of complex points $z=-i x=e^{-i \pi / 2} x, x>0$. Consequently,

$$
\begin{aligned}
-I_{2}= & \frac{1}{2 \pi \hbar} e^{-i \pi / 2} \int_{0}^{\infty} d x \exp \left\{\frac{i}{\hbar}[-(P-p-i \epsilon)(-i x)\right. \\
& \left.\left.+n \hbar \alpha \log \frac{x e^{-i \pi / 2}}{a}\right]\right\} .
\end{aligned}
$$

Transforming to a dimensionless variable $y=(P-p$ $-i \epsilon) x / \hbar$, the integral becomes

$$
\begin{aligned}
-I_{2}= & \frac{1}{2 \pi \hbar} \exp \left\{(n \alpha-i) \frac{\pi}{2}-i n \alpha \log [(P-p-i \epsilon) a / \hbar]\right\} \\
& \times \frac{\hbar}{P-p-i \epsilon} \int_{0}^{\infty} d y e^{-y} y^{i n \alpha} .
\end{aligned}
$$


Recognizing the remaining integral as a $\Gamma$ function of the complex argument $1+i n \alpha$,

$$
\begin{aligned}
-I_{2}= & \frac{\Gamma(1+i n \alpha)}{2 \pi(P-p-i \epsilon)} \exp \left\{(n \alpha-i) \frac{\pi}{2}\right. \\
& -i n \alpha \log [(P-p-i \epsilon) a / \hbar]\} .
\end{aligned}
$$

For $P<p$, we need to close the contour in the upper halfplane, but otherwise the procedure is analogous. Combining the two cases, we obtain a single analytic expression for the uniform transformation element,

$$
\begin{aligned}
\langle P \mid p\rangle_{n, \text { unif }}= & \frac{\Gamma(1+i n \alpha)}{2 \pi(|P-p|-i s \epsilon)} \exp \left[(n \alpha-i) \frac{\pi}{2} s\right. \\
& \left.-i n \alpha \log \frac{(|P-p|-i s \epsilon) a}{\hbar}\right],
\end{aligned}
$$

where $s=\operatorname{sgn}(P-p)$. As promised, the uniform (A4) and semiclassical (36) forms have identical dependence on $\hbar$ and $P-p$. The only difference lies in the dependence on $n \alpha$, in particular the semiclassical form is obtained if we keep only the first term in the asymptotic expansion of $\Gamma(1+$ in $\alpha)$ in $\langle P \mid p\rangle_{n, \text { unif }}$. However, for our conservative choice of $\alpha$ $\approx 33$ the agreement between $\langle P \mid p\rangle_{n, s c}$ and $\langle P \mid p\rangle_{n, \text { unif }}$ is such that they may be used interchangeably for any practical purposes.
[1] Surprisingly enough, softly chaotic systems resist semiclassical treatment often more than those with strongly chaotic behavior. It is because loop areas in phase space increase as chaos grows stronger. For a semiclassical analysis of a strongly chaotic system see, e.g., S. Tomsovic and E. J. Heller, Phys. Rev. E 47, 282 (1993); P. W. O'Connor, S. Tomsovic, and E. J. Heller, J. Stat. Phys. 68, 131 (1991). For a semiclassical analysis of a mixed system (with weak chaos) see, e.g., M. A. Sepúlveda and E. J. Heller, J. Chem. Phys. 101, 8004 (1994).

[2] W. H. Miller, J. Chem. Phys. 53, 3578 (1970).

[3] W. H. Miller, Adv. Chem. Phys. 25, 69 (1974).

[4] E. J. Heller, Prog. Theor. Phys. Suppl. N116, 45 (1994).

[5] G. F. Carrier, J. Fluid Mech. 24, 641 (1966).

[6] M. V. Berry, Proc. Phys. Soc. London 89, 479 (1966).

[7] We keep the discussion in terms of general canonical transformations although we often have in mind the semiclassical Van Vleck propagator when speaking of transformation elements.

[8] Even if the integral is not analytic, an Airy-function uniformization procedure can be used. See, e.g., [5,6].

[9] W. H. Miller and F. T. Smith, Phys. Rev. A 17, 939 (1978).

[10] L. M. Hubbard and W. H. Miller, J. Chem. Phys. 78, 1801 (1983).

[11] The small difference is the result of our neglecting the shift of SP points due to the finite extent of $\phi^{i}(p)$.

[12] H. Hoinkes, Rev. Mod. Phys. 52, 933 (1980).

[13] J. W. Strutt (Baron Rayleigh), Proc. R. Soc. London, Ser. A 79, 399 (1907).

[14] P. Beckmann and A. Spizzichino, The Scattering of Electromagnetic Waves from Rough Surfaces (Artech House, Norwood, 1987).

[15] N. Garcia and N. Cabrera, Phys. Rev. B 18, 576 (1978).

[16] R. F. Millar, Proc. Cambridge Philos. Soc. 69, 217 (1971).

[17] J. H. Van Vleck, Proc. Natl. Acad. Sci. U.S.A. 14, 178 (1928).

[18] M. C. Gutzwiller, Chaos in Classical and Quantum Mechanics (Springer, Berlin, 1990).

[19] The Kirchhoff diffraction method starts from the Weber integral representation (an analog of a more familiar Helmholtz representation in three dimensions)

$$
\psi\left(\mathbf{r}_{2}\right)=\int_{C} d s\left(\psi \frac{\partial}{\partial n} H_{1}^{(0)}(k R)-H_{1}^{(0)}(k R) \frac{\partial \psi}{\partial n}\right),
$$

where $R=\left|\mathbf{r}_{2}-\mathbf{r}\right|, \mathbf{r}$ is the coordinate vector of points on the closed curve $C$, and $\partial / \partial n$ is the derivative in the direction of an outward pointing normal vector $\hat{\mathbf{n}}$. For the derivation of Weber and Helmholtz representations, see, e.g., B. B. Baker and E. T. Copson, The Mathematical Theory of Huygens' Principle, 2nd ed. (Oxford University Press, London, 1950), p. 48; M. Born and E. Wolf, Principles of Optics, 6th ed. (Pergamon Press, Elmsford, NY, 1980), p. 375. The Kirchhoff method consists of approximating usually unknown values of $\psi$ and $\partial \psi / \partial n$ in the integrand. Originally, it was only used in the case of a plane screen with an aperture: Kirchhoff set $\psi$ and $\partial \psi / \partial n$ equal to zero on the dark side of the screen and equal to the unperturbed values $\left(\psi_{i n c}\right.$ and $\left.\partial \psi_{i n c} / \partial n\right)$ in the aperture. The term "Kirchhoff method" is now sometimes used for a more general approximation encompassing our example of the corrugated wall, whereby $\psi$ and $\partial \psi / \partial n$ at any point of the surface are approximated by the values that would be present on the tangent plane at that point. See, e.g., The Scattering of Electromagnetic Waves from Rough Surfaces, Ref. [14], p. 20.

[20] U. Garibaldi, A. C. Levi, R. Spaldacini, and G. E. Tommei, Surf. Sci. 48, 649 (1975).

[21] M. V. Berry and E. Bodenschatz, J. Mod. Opt. 46, 349 (1999).

[22] This remarkable effect appears also in our case (for $\psi_{\text {scat }}$ only) when $k_{1 x}=0$ : Note that then the paraxial approximation

$$
k_{2 y, n}=\sqrt{k^{2}-(\beta n)^{2}} \approx k-\frac{\beta^{2} n^{2}}{2 k}
$$

(valid for $\epsilon \ll 1$ ) implies that the scattered wave in both the exact and RM solutions has the property

$$
\psi_{\text {scat }}\left(x-\frac{\pi}{\beta}, y+y_{T}\right)=\psi_{\text {scat }}(x, y) e^{i k y_{T}},
$$

where $y_{T}=2 \pi k / \beta^{2}$ is the Talbot distance. 